\title{
Intelligent Reflecting Surfaces: Physics, Propagation, and Pathloss Modeling
}

\author{
Özgecan Özdogan, Student Member, IEEE, Emil Björnson, Senior Member, IEEE, Erik G. Larsson, Fellow, IEEE
}

\begin{abstract}
Intelligent reflecting surfaces can improve the communication between a source and a destination. The surface contains metamaterial that is configured to "reflect" the incident wave from the source towards the destination. Two incompatible pathloss models have been used in prior work. In this letter, we derive the far-field pathloss using physical optics techniques and explain why the surface consists of many elements that individually act as diffuse scatterers but can jointly beamform the signal in a desired direction with a certain beamwidth. We disprove one of the previously conjectured pathloss models.
\end{abstract}

Index Terms-Intelligent reflecting surface, pathloss model.

\section{INTRODUCTION}

Conventional wireless communication systems consist of a transmitter sending information-bearing electromagnetic waves to a receiver via an uncontrollable propagation environment. When searching for beyond 5G network architectures, there is a growing interest in creating real-time reconfigurable propagation environments [1]-[4]. This can potentially be achieved by deploying special surfaces, known as intelligent reflecting surfaces (IRS) [1], software-controlled metasurfaces [2]-[4], and reconfigurable intelligent surfaces [5], [6], that can control how the waves that reach them are reflected. In this context, the word "reflection" has a wide meaning [7], including diffuse reflection (e.g., scattering by rough material) and ideal specular reflection (e.g., from an infinite mirror).

While the design of reconfigurable surfaces has a long history in the electromagnetic literature [8], the communication analysis is in its infancy. There is no consensus on the basic propagation modeling, but two incompatible pathloss models have been conjectured without derivation from physical principles: surfaces consisting of many scattering elements [9], [10] and surfaces consisting of many ideal mirrors [5]. In this letter, we fill this gap by first explaining in Section $[1$ how a passive metallic surface scatters an incident wave and then derive in Section III how an IRS must be designed to mimic such a surface while also controlling the directivity of the scattered wave. This results in a rigorous pathloss model and a way to build system models that can be used for further research.

\section{Preliminaries: Passive Metallic Surface}

In this section, we review preliminaries on the field strength and beamwidth of the waveform scattered by a "passive" metallic (perfectly conducting) plate of finite size. The results will later be used to explain the ideal operation of an IRS.

The letter was supported by ELLIIT and the Swedish Research Council.

The authors are with the Department of Electrical Engineering (ISY), Linköping University, SE-58183 Linköping, Sweden. Email: \{ozgecan.ozdogan,emil.bjornson,erik.g.larsson\}@ liu.se.

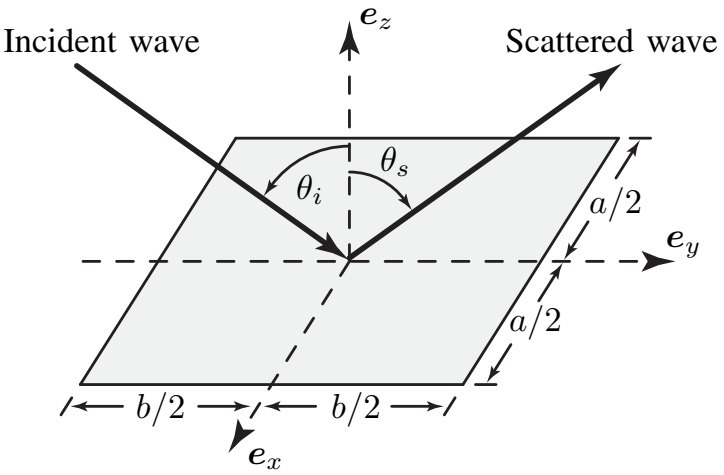

Fig. 1: An incident wave is scattered by a $a \times b$ metal plate.

We consider a rectangular, perfectly conducting plate of size $a \times b$, and negligible thickness, located in the horizontal plane (spanned by $\boldsymbol{e}_{x}, \boldsymbol{e}_{y}$ ). A point source far away, at distance $d_{i}$, is radiating a linearly polarized electromagnetic wave with wave number $k=\frac{2 \pi}{\lambda}$ where $\lambda$ is the wavelength. We assume, for the sake of argument, that the polarization of the source is such that the E-field is parallel to $\boldsymbol{e}_{x}$ and the H-field lies in the plane spanned by $\boldsymbol{e}_{y}$ and $\boldsymbol{e}_{z}$. Let $\theta_{i} \in\left[0, \frac{\pi}{2}\right]$ denote the angle of incidence, that is, the angle between the Poynting vector of the wave and $\boldsymbol{e}_{z}$. This setup is illustrated in Fig. 1

We further assume that $d_{i}$ is sufficiently large relative to $a$ and $b$ (i.e., the source is in the far-field) that the curvature of the wavefront, over the dimensions of the plate, can be neglected. Hence, the impinging wavefield is approximated as a plane wave with some magnitude $E_{i}$. To determine when this approximation holds, we compute the phase differential between a spherical wave and its plane-wave approximation between the center and the edges of the plate. For the sake of argument, suppose the plate is oriented such that the Poynting vector enters with $\theta_{i}=0$ at the plate center; however the analysis can be generalized to arbitrary incidence angles. With the plane-wave approximation, the wave from the source travels a distance of $d_{i}$ to any point on the plate, but the wave is actually spherical and at the edges of the plate it has traveled the distance $\sqrt{d_{i}^{2}+\frac{b^{2}}{4}}$. The phase discrepancy is

$$
k\left(\sqrt{d_{i}^{2}+\frac{b^{2}}{4}}-d_{i}\right) \approx \frac{\pi}{4} \frac{b^{2}}{\lambda d_{i}}
$$

With e.g., $b=1 \mathrm{~m}, d_{i}=100 \mathrm{~m}, \lambda=0.1 \mathrm{~m}$, this is less than five degrees and should have a minor impact. The incident plane wave has the electric and magnetic field distributions

$$
\begin{aligned}
& \mathbf{E}_{i}=E_{i} e^{-j k\left(\sin \left(\theta_{i}\right) y-\cos \left(\theta_{i}\right) z\right)} \boldsymbol{e}_{x} \\
& \mathbf{H}_{i}=-\frac{E_{i}}{\eta}\left(\cos \left(\theta_{i}\right) \boldsymbol{e}_{y}+\sin \left(\theta_{i}\right) \boldsymbol{e}_{z}\right) e^{-j k\left(\sin \left(\theta_{i}\right) y-\cos \left(\theta_{i}\right) z\right)}
\end{aligned}
$$


where $\eta$ is the characteristic impedance of the medium.

The E-field will induce motion of electrons in the plate. The electrons will move in the direction of $\boldsymbol{e}_{x}$, but not in $\boldsymbol{e}_{y}$ (since the E-field is orthogonal to $e_{y}$ ) and also not in the $e_{z}$-direction since the plate is assumed thin. The moving electrons induce electromagnetic radiation, resulting in a scattered wave.

Lemma 1. The squared magnitude of the scattered field, in the $\boldsymbol{e}_{y}, \boldsymbol{e}_{z}$ plane and at an arbitrary observation angle $\theta_{s} \in\left[0, \frac{\pi}{2}\right]$ (measured against $\boldsymbol{e}_{z}$ ) is

$$
S\left(r, \theta_{s}\right)=\left(\frac{a b}{\lambda}\right)^{2} \frac{E_{i}^{2}}{r^{2}} \cos ^{2}\left(\theta_{i}\right)\left(\frac{\sin \left(\frac{\pi b}{\lambda}\left(\sin \left(\theta_{s}\right)-\sin \left(\theta_{i}\right)\right)\right)}{\frac{\pi b}{\lambda}\left(\sin \left(\theta_{s}\right)-\sin \left(\theta_{i}\right)\right)}\right)^{2}
$$

at a far-field observation distance $r \geq \frac{2 \max \left(a^{2}, b^{2}\right)}{\lambda}$.

Proof: This result follows from standard physical optics techniques (which neglect edge effects) as in [11, Example 11-3].

The expression in (4) for the magnitude of the scattered field in the far-field has several intuitive properties; for example, it is proportional to the area $(a b)^{2}$ of the plate and to $E_{i}^{2} \propto$ $1 / d_{i}^{2}$, since the impinging wave from a point source in lineof-sight $(\mathrm{LoS})$ has a field strength inversely proportional to $d_{i}^{2}$. As expected from Snell's law, for the polarization that we consider, $S\left(r, \theta_{s}\right)$ attains its maximum for the observation angle $\theta_{s}=\theta_{i}$, which is the specular direction, for which the last parenthesis in (4) is unity 1

\section{A. Beamwidth of the Scattered Wave}

The expression $S\left(r, \theta_{s}\right)$ reveals that the scattered field looks like a beam that tapers off as $\theta_{s}$ is moved away from $\theta_{i}$. The $3-\mathrm{dB}$ beamwidth equals twice the deviation $\left|\theta_{s}-\theta_{i}\right|$ required to make the square of the second parenthesis in (4) equal to $1 / 2$. Using the Taylor expansion $\cos (x)=1+O\left(x^{2}\right)$ and standard trigonometric identities, we have

$$
\begin{aligned}
& \sin \left(\theta_{s}\right)-\sin \left(\theta_{i}\right)=\sin \left(\theta_{i}+\theta_{s}-\theta_{i}\right)-\sin \left(\theta_{i}\right) \\
& =\sin \left(\theta_{i}\right) \cos \left(\theta_{s}-\theta_{i}\right)+\cos \left(\theta_{i}\right) \sin \left(\theta_{s}-\theta_{i}\right)-\sin \left(\theta_{i}\right) \\
& =\cos \left(\theta_{i}\right)\left(\theta_{s}-\theta_{i}\right)+O\left(\left(\theta_{s}-\theta_{i}\right)^{2}\right) .
\end{aligned}
$$

From (5) and using the Taylor expansions $\frac{\sin (x)}{x}=1-\frac{x^{2}}{6}+$ $O\left(x^{3}\right)$ and $\left(\frac{\sin (x)}{x}\right)^{2}=1-\frac{x^{2}}{3}+O\left(x^{3}\right)$, we obtain the following second-order approximation of (4):

$$
\begin{aligned}
S\left(r, \theta_{s}\right)= & \left(\frac{a b}{\lambda}\right)^{2} \frac{E_{i}^{2}}{r^{2}} \cos ^{2}\left(\theta_{i}\right)\left(1-\frac{\pi^{2} b^{2}}{\lambda^{2}} \frac{\cos ^{2}\left(\theta_{i}\right)\left(\theta_{s}-\theta_{i}\right)^{2}}{3}\right) \\
& +O\left(\left(\theta_{s}-\theta_{i}\right)^{3}\right) .
\end{aligned}
$$

Hence, the 3-dB beamwidth is approximately twice the deviation $\left|\theta_{s}-\theta_{i}\right|$ required to make $\frac{\pi^{2} b^{2}}{\lambda^{2}} \frac{\cos ^{2}\left(\theta_{i}\right)\left(\theta_{s}-\theta_{i}\right)^{2}}{3}=\frac{1}{2}$ :

$$
\left|\theta_{s}-\theta_{i}\right|<\sqrt{\frac{1}{2} \frac{3 \lambda^{2}}{\pi^{2} b^{2} \cos ^{2}\left(\theta_{i}\right)}}=\sqrt{\frac{3}{2} \frac{\lambda}{\pi b \cos \left(\theta_{i}\right)}} .
$$

\footnotetext{
${ }^{1}$ For other incident wave polarizations, this is true only approximately. However, the observation angle that maximizes $S\left(r, \theta_{s}\right)$ approaches $\theta_{i}$ as the size of the plate increases.
}

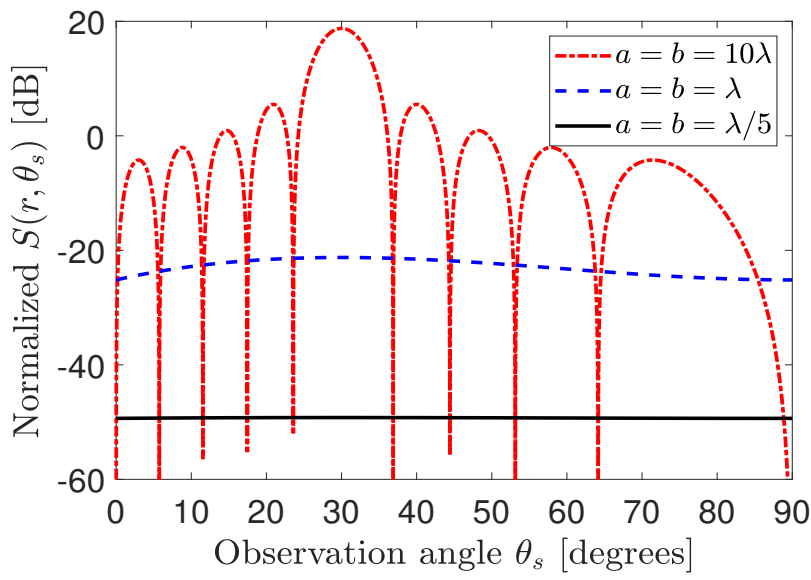

Fig. 2: Normalized squared magnitude of the scattered field as a function of the angle $\theta_{s}$ where $\theta_{i}=30^{\circ}$.

This inequality shows that the $3-\mathrm{dB}$ beamwidth is inversely proportional to the plate width $b$. The beamwidth is also proportional to the wavelength $\lambda$, thus a fixed-size plate can provide an extremely narrow beamwidth in the visible spectrum (i.e., an almost perfect specular reflection), but 45 orders-of-magnitude wider beamwidths in the typical radio spectrum bands ${ }^{2}$

The plate acts as a mirror in the sense that the diffusely reflected or, more accurately, the scattered field has a 3-dB beamwidth inversely proportional to $b$. Hence, it becomes very small if $b$ is large relative to $\lambda$. This scaling is no surprise: if we think of the plate as an antenna, it is just the usual relation that beamwidth is inversely proportional to antenna aperture.

Fig. 2] shows the squared magnitude $S\left(r, \theta_{s}\right)$ as a function of $\theta_{s}$. It is normalized by multiplying with $\frac{r^{2}}{E_{i}^{2}}$, and we consider $\theta_{i}=30^{\circ}$. When $a$ and $b$ are smaller or equal to the wavelength, the scattered field is almost equally strong in all observation angles. It is first when the plate is substantially larger than $\lambda$ that the beamwidth becomes small. The $3-\mathrm{dB}$ beamwidth is $\approx 5.7^{\circ}$ for a plate with $a=b=10 \lambda$ where the second-order approximation in (7) gives $\approx 5.2^{\circ}$.

Corollary 1. A receiving antenna of effective electrical size $\frac{\lambda}{v} \times \frac{\lambda}{v}$, located at distance $r \gg b / v$ from the plate with angle $\theta_{s}$ to the antenna center will receive the signal power

$$
S\left(r, \theta_{s}\right)\left(\frac{\lambda}{v}\right)^{2}
$$

Proof: The antenna will see the plate through an angular window of $\frac{\lambda}{v r}$ radians. As long as $\frac{\lambda}{v r} \ll \frac{\lambda}{b \cos \left(\theta_{i}\right)}$, i.e., approximately, $r \gg b / v$, the field strength will be approximately constant over the antenna and (8) is obtained.

Since $E_{i}^{2} \propto 1 / d_{i}^{2}$ in LoS, Corollary 1 proves that the received power is proportional to $(a b)^{2} /\left(d_{i} r\right)^{2}$, where the proportionality constant depends on the wavelength and angles. If $\theta_{s}=\theta_{i}$, the received power increases monotonically with $a$ and $b$, because more energy is induced into the plate and then radiated in a gradually narrower beam. Even when the

\footnotetext{
${ }^{2}$ Comparing radio signals at $6 \mathrm{GHz}$ with green visible light at $600 \mathrm{THz}$, the former has a $10^{5}$ larger wavelength and thus needs a $10^{5}$ wider plate to give a reflection with the same beamwidth as for green light.
} 
beamwidth is small in absolute terms, it can be large relative to $\lambda / r$ and, thus, most of the energy of the scattered field anyway misses the receiver antenna aperture. This is why the received power in (8) is proportional to $1 / r^{2}$. As $a$ and $b$ are increased without bound, eventually the plane-wave approximation of the incident field breaks down and the results in this section become inaccurate. In the asymptotic limit, geometric optics can be used instead to model a perfect mirror.

\section{B. Multiple Adjacent Metallic Surfaces}

Since the plate has a finite size, we can deploy multiple adjacent plates. If the gaps in between are sufficiently large then coupling effects can be neglected and superposition applies. When the scattered fields from these plates are received at a given location, the relative phase shifts will lead to constructive or destructive interference. Under ideal constructive interference, the squared field strength from $N$ plates will be

$$
\left(N \sqrt{S\left(r, \theta_{s}\right)}\right)^{2}=N^{2} S\left(r, \theta_{s}\right) .
$$

The variables $N, a$, and $b$ only appear in (9) as a joint term $(N a b)^{2}$, where $N a b$ is the total area of the $N$ plates. Hence, it does not matter if the total area is made up by many small or a few large plates, the maximum received power is the same.

\section{System Model FOR Intelligent Metasurfaces}

If the incident angle $\theta_{i}$ from the transmitter to the surface equals the observation angle $\theta_{s}$ leading to the receiver, then the passive surface considered in Section $\Pi$ represents the ideal case. Since the angles are generally different when one of the devices is mobile, the main purpose of an IRS is to achieve "anomalous reflection" [7], which means shaping the scattered field so that the main beam is directed towards the receiver.

We now consider an IRS consisting of a metasurface of the same dimensions as in Fig. 1 and the same impinging plane wave. The goal of an IRS is to achieve total reflection with its main beam pointing in a desired direction that we denote as $\theta_{r}$. Hence, the surface must be designed to redirect the incident plane wave $\left(\mathbf{E}_{i}, \mathbf{H}_{i}\right)$ in (2) and obtain the following ideal field distributions of the reflected/scattered wave:

$\mathbf{E}_{r}=E_{r} e^{-j k\left(\sin \left(\theta_{r}\right) y+\cos \left(\theta_{r}\right) z\right)} \boldsymbol{e}_{x}$,

$\mathbf{H}_{r}=-\frac{E_{r}}{\eta}\left(\sin \left(\theta_{r}\right) \boldsymbol{e}_{z}-\cos \left(\theta_{r}\right) \boldsymbol{e}_{y}\right) e^{-j k\left(\sin \left(\theta_{r}\right) y+\cos \left(\theta_{r}\right) z\right)}$.

A widely used approach in the literature that designs reflective metasurfaces is based on the generalized Snell's law of reflection [12]. Using this method, the required surface phase profile to transform the incident wave $\left(\mathbf{E}_{i}, \mathbf{H}_{i}\right)$ into $\left(\mathbf{E}_{r}, \mathbf{H}_{r}\right)$ is obtained by tailoring the surface impedance. At the surface $(z=0)$, the superposition of the incident and reflected E-field can be written as [13]

$$
\mathbf{E}_{t}=E_{i} e^{-j k \sin \left(\theta_{i}\right) y} \boldsymbol{e}_{x}+E_{r} e^{-j k \sin \left(\theta_{r}\right) y} \boldsymbol{e}_{x} .
$$

Then, the desired phase of the desired reflection coefficient is

$$
\phi_{r}(y)=\angle\left(\frac{E_{r} e^{-j k \sin \left(\theta_{r}\right) y}}{E_{i} e^{-j k \sin \left(\theta_{i}\right) y}}\right)=-k \sin \left(\theta_{r}\right) y+k \sin \left(\theta_{i}\right) y
$$

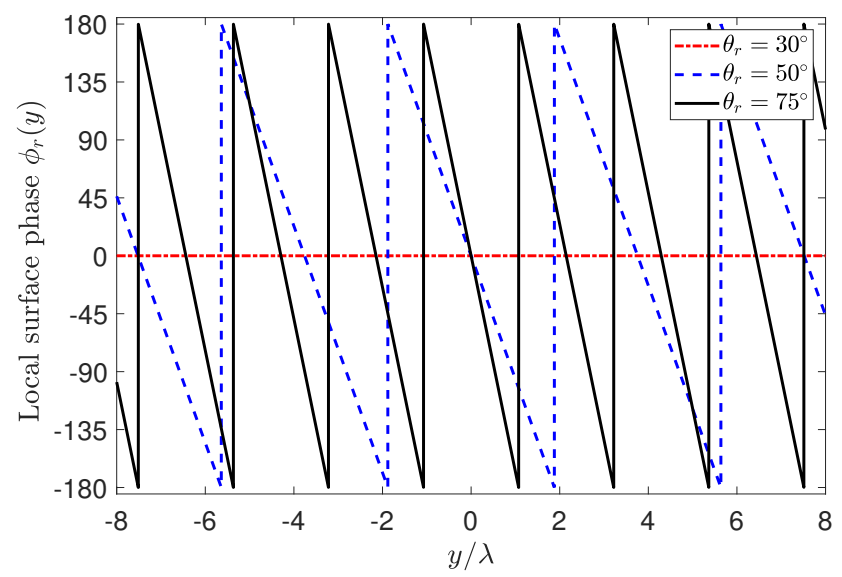

Fig. 3: Local surface phase that is required to redirect the incident wave with $\theta_{i}=30^{\circ}$ in a desired direction $\theta_{r}$.

and differentiating it with respect to $y$ gives the gradient of the reflection coefficient in the generalized Snell's law:

$$
k\left(\sin \left(\theta_{i}\right)-\sin \left(\theta_{r}\right)\right)=\frac{d \phi_{r}(y)}{d y},
$$

which gives the relation between $\theta_{i}, \theta_{r}$ and the local surface phase $\phi_{r}(y)$. By altering the surface impedance, $\phi_{r}(y)$ is obtained at each point of the surface and the output wave's desired phase $-k \sin \left(\theta_{i}\right) y+\phi_{r}(y)=-k \sin \left(\theta_{r}\right) y$ is achieved.

Fig. 3 shows the required local surface phase profile to redirect $\left(\mathbf{E}_{i}, \mathbf{H}_{i}\right)$ with $\theta_{i}=30^{\circ}$ into $\left(\mathbf{E}_{r}, \mathbf{H}_{r}\right)$ for different values of $\theta_{r}$. Fig. 3 shows that it is easier to implement a surface when the desired $\theta_{r}$ is close to $\theta_{i}$ since the required phase profile varies more slowly over the surface (i.e., 14 is closer to zero). In practical implementations, the desired local phase shift in the metasurface is discretized by dividing the surface into sub- $\lambda$-sized elements, each having a constant phase shift. The smaller the elements are, the more closely the local phase shift can be approximated. In [14], the phase distribution is discretized/quantized with a step size of $\lambda / 5$, to represent the finite number of resonators used in practice. In [15], the surface is discretized by $\lambda / 8$ as also shown in Fig. 4

Small high-precision elements require sophisticated design and expensive hardware, and also lead to coupling issues. On the other hand, if the elements are too large (relative to $\lambda$ ) then the required local phase will be coarsely quantized. This will lead to a mismatch between the desired reflection angle and the surface response. In this paper, we neglect the errors due to quantization.

\section{A. Propagation and Pathloss Model}

In contrast to a metallic surface, for which it does not matter if it consists of one or multiple plates (see Section II-B, an IRS must consist of many small elements to reconfigure the local phases with high resolution and thereby achieves a main beam with the desired angle $\theta_{r}$. This has no negative impact on the maximum magnitude of the scattered field and the beamwidth will not be narrower than in the ideal case of a passive metallic surface. As discussed in Section III, the incoming wave's E-field in (2) induces an electric surface current in the direction of $e_{x}$. This current is adjusted in the 


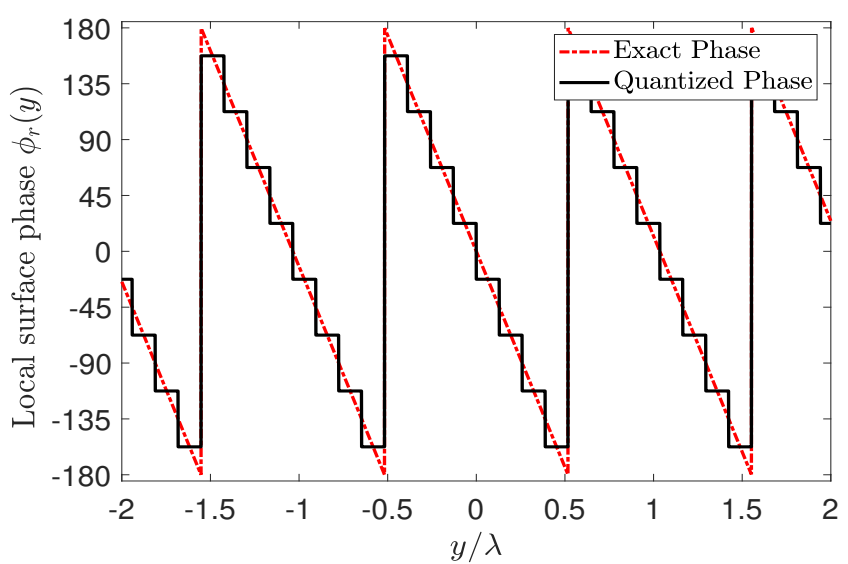

Fig. 4: The quantized local surface phase that is required to redirect the incident wave with $\theta_{i}=0^{\circ}$ to $\theta_{r}=75^{\circ}$.

IRS by tuning the surface impedance in each element to obtain a surface phase profile that approximates that required by the generalized Snell's law. This operation results in a scattered wave with maximum amplitude towards $\theta_{r}$ instead of $\theta_{i}$.

Lemma 2. When using an IRS to reflect a signal in the direction $\theta_{r}$, the squared magnitude of the scattered field at an arbitrary observation angle $\theta_{s} \in\left[-\frac{\pi}{2}, \frac{\pi}{2}\right]$ is

$$
\begin{aligned}
& S_{\mathrm{IRS}}\left(r, \theta_{s}, E_{i}^{2}\right) \\
& =\left(\frac{a b}{\lambda}\right)^{2} \frac{E_{i}^{2} \cos ^{2}\left(\theta_{i}\right)}{r^{2}}\left(\frac{\sin \left(\frac{\pi b}{\lambda}\left(\sin \left(\theta_{s}\right)-\sin \left(\theta_{r}\right)\right)\right)}{\frac{\pi b}{\lambda}\left(\sin \left(\theta_{s}\right)-\sin \left(\theta_{r}\right)\right)}\right)^{2}
\end{aligned}
$$

at a far-field distance $r \geq \frac{2 \max \left(a^{2}, b^{2}\right)}{\lambda}$.

Proof: The negligible thickness of the surface allows us to write the electric current density on the surface $\left(z=0, y=y^{\prime}\right)$ approximately as $J_{x}=\frac{2 E_{i}}{\eta} \cos \left(\theta_{i}\right) e^{-j k \sin \left(\theta_{r}\right) y^{\prime}}[11$, Eq. (754)] assuming the surface is lossless, i.e., $E_{i}^{2} \cos \left(\theta_{i}\right)=$ $E_{r}^{2} \cos \left(\theta_{r}\right)$. Then, we use the same steps as in Lemma 1

The intercepted power by the IRS is the same as for the perfectly conducting plate in Section III but the maximum of $S_{\text {IRS }}\left(r, \theta_{s}, E_{i}^{2}\right)$ is achieved at $\theta_{s}=\theta_{r}$ instead of $\theta_{s}=\theta_{i}$.

Suppose the transmit power is $P_{t}$ and the transmitter has antenna gain $G_{t}$. Then the relation between $E_{i}$ and $P_{t}$ is

$$
\frac{E_{i}^{2}}{2 \eta}=\frac{P_{t} G_{t}}{4 \pi d_{i}^{2}}
$$

where $E_{i}$ has unit Volt $/ \mathrm{m}$ and $\eta \approx 377$ Ohm. Furthermore, assume that the effective area of the receiver antenna is $\frac{\lambda^{2}}{4 \pi} G_{r}$, where $G_{r}$ is the antenna gain. Then, the received signal power $P_{r}$ for a receiver at far-field distance $r$ in direction $\theta_{s}$ is

$$
P_{r}\left(P_{t}, d_{i}, r, \theta_{s}\right)=\frac{1}{2 \eta} S_{\text {IRS }}\left(r, \theta_{s}, \frac{P_{t} G_{t} \eta}{2 \pi d_{i}^{2}}\right)\left(\frac{\lambda^{2}}{4 \pi} G_{r}\right) .
$$

Corollary 2. When using an IRS to reflect a signal in the direction $\theta_{r}$, the pathloss at the far-field distance $r$ is

$$
\begin{aligned}
& \beta_{\mathrm{IRS}}\left(r, d_{i}, \theta_{s}\right)=\frac{P_{r}\left(P_{t}, d_{i}, r, \theta_{s}\right)}{P_{t}} \\
& =\frac{G_{t} G_{r}}{(4 \pi)^{2}}\left(\frac{a b}{d_{i} r}\right)^{2} \cos ^{2}\left(\theta_{i}\right)\left(\frac{\sin \left(\frac{\pi b}{\lambda}\left(\sin \left(\theta_{s}\right)-\sin \left(\theta_{r}\right)\right)\right)}{\frac{\pi b}{\lambda}\left(\sin \left(\theta_{s}\right)-\sin \left(\theta_{r}\right)\right)}\right)^{2}
\end{aligned}
$$

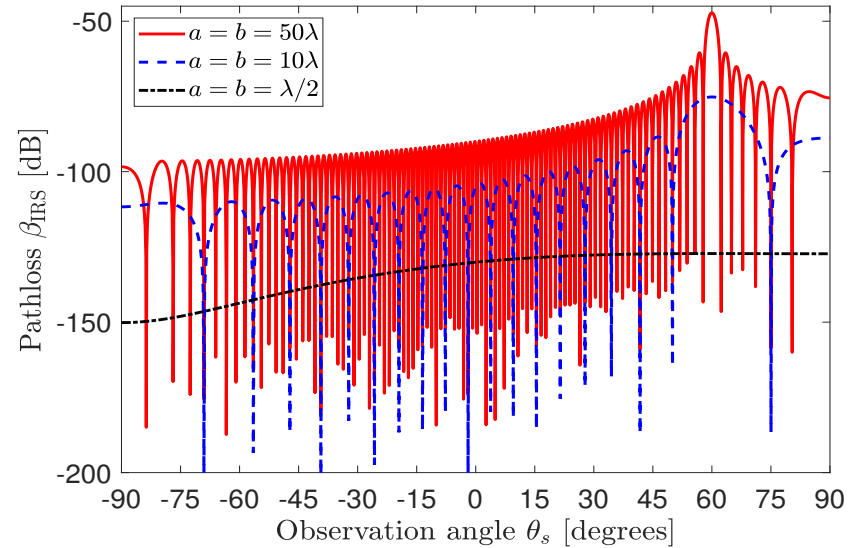

Fig. 5: The pathloss of the reflected path. The angles are $\theta_{i}=$ $30^{\circ}$ and $\theta_{r}=60^{\circ}$ where the antenna gains are $G_{t}=G_{r}=5$ $\mathrm{dB}$, the distances are $d_{i}=50$ and $r=25$ meters.

and in the ideal case when the receiver has $\theta_{s}=\theta_{r}$, the pathloss expression simplifies to

$$
\beta_{\mathrm{IRS}}\left(r, d_{i}, \theta_{r}\right)=\frac{G_{t} G_{r}}{(4 \pi)^{2}}\left(\frac{a b}{d_{i} r}\right)^{2} \cos ^{2}\left(\theta_{i}\right) .
$$

Remark 1. The derivations have assumed the incoming wave and the desired scattered wave have E-fields that are both parallel to $e_{x}$, but the analysis can be generalized. The end result will still become the pathloss expression $\beta_{\mathrm{IRS}}\left(r, d_{i}, \theta_{r}\right)$ that only depends on the total effective area $a b \cos \left(\theta_{i}\right)$ of the IRS as seen from the transmitter.

Fig. 5 shows the pathloss $\beta_{\text {IRS }}\left(r, d_{i}, \theta_{s}\right)$ as a function of $\theta_{s}$ for different sizes of the IRS and $\theta_{r}=60^{\circ}$. The maximum is achieved at $\theta_{s}=\theta_{r}$ and the main beamwidth gets narrower as the IRS's surface area increases. When the dimension is subwavelength $(\leq \lambda / 2)$, the IRS almost acts as a diffuse scatterer.

\section{B. Interpreting an IRS as an Array of Diffuse Scatterers}

As noted above, an IRS of size $a \times b$ consists of many sub$\lambda$-sized surface elements. Suppose the IRS consists of $N_{a} \times N_{b}$ elements, each having the size $\frac{a}{N_{a}} \times \frac{b}{N_{b}}$, where $\frac{a}{N_{a}}, \frac{b}{N_{b}} \leq \lambda$. The pathloss between the transmitter and receiver through the $n^{\text {th }}$ surface element (assuming the others are removed) is

$$
\beta_{\mathrm{IRS}}^{s}\left(r, d_{i}, \theta_{r}\right)=\frac{G_{t} G_{r}}{(4 \pi)^{2}}\left(\frac{a b}{N_{a} N_{b} d_{i} r}\right)^{2} \cos ^{2}\left(\theta_{i}\right)
$$

since the last term in 18 is approximately unity for an element of this size (as shown in Fig. 5). Note that $\beta_{\mathrm{IRS}}^{s}\left(r, d_{i}, \theta_{r}\right)$ is the same for all $n$ since we have assumed $r \geq \frac{2 \max \left(a^{2}, b^{2}\right)}{\lambda}$.

Let $\phi_{n}$ denote the local surface phase of the $n^{\text {th }}$ element. If it is selected to achieve constructive interference from all $N=$ $N_{a} N_{b}$ surface elements at the receiver, the pathloss between the transmitter and receiver through the whole IRS is

$$
\left(N \sqrt{\beta_{\mathrm{IRS}}^{s}\left(r, d_{i}, \theta_{r}\right)}\right)^{2}=\beta_{\mathrm{IRS}}\left(r, d_{i}, \theta_{s}\right) .
$$

Hence, we can interpret an IRS as an array of diffuse scatterers (each sub- $\lambda$-sized) that phase-align their reflected signals at the receiver and thereby achieve "anomalous" reflection. 


\section{System Model for IRS-Supported Communications}

To exemplify how one can derive a physically correct system model for IRS-supported communication, we consider an LoS setup where $\sqrt{\beta_{\mathrm{sd}}} e^{j \phi_{\mathrm{sd}}}$ is the direct channel between the single-antenna source and destination. When including the reflected path from the IRS, we get the received signal

$$
y=\left(\sqrt{\beta_{\mathrm{IRS}}^{s}} \mathbf{h}_{\mathrm{sr}}^{\mathrm{T}} \boldsymbol{\Phi} \mathbf{h}_{\mathrm{rd}}+\sqrt{\beta_{\mathrm{sd}}} e^{j \phi_{\mathrm{sd}}}\right) x+w
$$

where $\mathbf{h}_{\mathrm{sr}}=\left[e^{j \psi_{1}^{\mathrm{sr}}}, \ldots, e^{j \psi_{n}^{\mathrm{sr}}}, \ldots, e^{j \psi_{N}^{\mathrm{sr}}}\right]^{\mathrm{T}}$ and $\mathbf{h}_{\mathrm{rd}}=$ $\left[e^{j \psi_{1}^{\mathrm{rd}}}, \ldots, e^{j \psi_{n}^{\mathrm{rd}}}, \ldots, e^{j \psi_{N}^{\mathrm{rd}}}\right]^{\mathrm{T}}$ are the normalized LoS channels between the source and IRS and the IRS and receiver, respectively. The signal $x$ has power $P_{t}, w \sim \mathcal{N}_{\mathbb{C}}\left(0, \sigma^{2}\right)$ is additive noise, and the surface phases of each surface element are stacked in $\boldsymbol{\Phi}=\operatorname{diag}\left(e^{j \phi_{1}}, \ldots, e^{j \phi_{n}}, \ldots, e^{j \phi_{N}}\right)$, which is a diagonal matrix. An equivalent way to write 22 is

$$
y=\sqrt{\beta_{\mathrm{IRS}}^{s}} \sum_{n=1}^{N} e^{j\left(\psi_{n}^{\mathrm{sr}}+\psi_{n}^{\mathrm{rd}}+\phi_{n}\right)} x+\sqrt{\beta_{\mathrm{sd}}} e^{j \phi_{\mathrm{sd}}} x+w .
$$

The IRS can select $\boldsymbol{\Phi}$ to maximize the received signal power [9]. If we select $\phi_{n}=\phi_{\text {sd }}-\psi_{n}^{\mathrm{sr}}-\psi_{n}^{\text {rd }}$ to phase-align all the signal terms in 23, we obtain $y=\left(N \sqrt{\beta_{\mathrm{IRS}}^{s}}+\sqrt{\beta_{\mathrm{sd}}}\right) e^{j \phi_{\mathrm{sd}}} x+w$ and the signal-to-noise ratio (SNR) is

$$
\mathrm{SNR}=\frac{\left(N \sqrt{\beta_{\mathrm{IRS}}^{s}}+\sqrt{\beta_{\mathrm{sd}}}\right)^{2} P_{t}}{\sigma^{2}}=\frac{\left(\sqrt{\beta_{\mathrm{IRS}}}+\sqrt{\beta_{\mathrm{sd}}}\right)^{2} P_{t}}{\sigma^{2}}
$$

where the second equality follows from (21). Hence, the considered phase-shifts coincide with those achieved by the discretized generalized Snell's law, with the extra condition that not only the $N$ terms in the sum in (23) are phase-aligned, but that they are also phase-aligned with the LoS path.

If an IRS is used for "anomalous" reflection, there is no need to take the detour via 22, but we use the IRS's total pathloss in 190 and directly write the received signal as $y=$

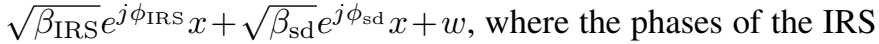
elements have already been aligned. It then only remains to select the common phase $\phi_{\text {IRS }}$ of all IRS elements to equal $\phi_{\text {sd }}$. However, there are other scenarios (e.g., with multiple antennas) where one can start from a system model as (22).

\section{SUMmARY AND RELATION TO PRIOR WORK}

We have used physical optics techniques to derive the pathloss expression in (19) for an IRS that is configured to reflect an incoming wave from a far-field source towards a receiver in the far-field. Even if the incoming signal is a plane wave, the reflected signal is a beam with beamwidth inversely proportional to the size of the IRS. Importantly, the received signal power is proportional to the square of the IRS area and to $1 /\left(d_{i} r\right)^{2}$, where $d_{i}$ is the distance between the transmitter and IRS, and $r$ is the distance between the IRS and receiver. This disproves the conjecture in [5] that the received power would be proportional to $1 /\left(d_{i}+r\right)^{2}$. That conjecture might hold for an infinitely large IRS acting or in the near-field, but provably not in the far-field setup studied herein ${ }^{3}$ In particular, one cannot use multiple infinite-sized IRS as in [5]. However, the system models used in [6], [9], [10] are essentially correct, if the pathloss of each element are selected according to 20.

A practical IRS consists of $N$ sub-wavelength-sized elements that scatter the incoming signals with unique phaseshifts to achieve coherent beamforming in a direction of interest. This is rather similar to a phased array, except that the signal power comes from another place. The ideal phaseshifts that create a single beam are given by the generalized Snell's law, but if a superposition of multiple beams should be created, the phase-shifts must be explicitly optimized.

\section{REFERENCES}

[1] Q. Wu and R. Zhang, "Towards smart and reconfigurable environment: Intelligent reflecting surface aided wireless network," CoRR, vol. abs/1905.00152, 2019.

[2] C. Liaskos, S. Nie, A. Tsioliaridou, A. Pitsillides, S. Ioannidis, and I. Akyildiz, "A new wireless communication paradigm through softwarecontrolled metasurfaces," IEEE Commun. Mag., vol. 56, no. 9, pp. 162 169, 2018.

[3] E. Björnson, L. Sanguinetti, H. Wymeersch, J. Hoydis, and T. L. Marzetta, "Massive MIMO is a reality-What is next? Five promising research directions for antenna arrays," Digital Signal Processing, vol. 94, pp. 3-20, Nov. 2019.

[4] M. D. Renzo et al., "Smart radio environments empowered by reconfigurable AI meta-surfaces: an idea whose time has come," EURASIP Journal on Wireless Commun. and Networking, vol. 2019:129, 2019.

[5] E. Basar, M. Di Renzo, J. De Rosny, M. Debbah, M. Alouini, and R. Zhang, "Wireless communications through reconfigurable intelligent surfaces," IEEE Access, vol. 7, pp. 116753-116773, 2019.

[6] C. Huang, A. Zappone, G. C. Alexandropoulos, M. Debbah, and C. Yuen, "Reconfigurable intelligent surfaces for energy efficiency in wireless communication," IEEE Transactions on Wireless Communications, vol. 18, no. 8, pp. 4157-4170, Aug 2019.

[7] L. Liang, M. Qi, J. Yang, X. Shen, J. Zhai, W. Xu, B. Jin, W. Liu, Y. Feng, C. Zhang, H. Lu, H.-T. Chen, L. Kang, W. Xu, J. Chen, T. J. Cui, P. Wu, and S. Liu, "Anomalous terahertz reflection and scattering by flexible and conformal coding metamaterials," Advanced Optical Materials, vol. 3, no. 10, pp. 1374-1380, 2015.

[8] J. Shaker, M. Chaharmir, and J. Ethier, Reflectarray Antennas: Analysis, Design, Fabrication, and Measurement. Artech House, 2014.

[9] Q. Wu and R. Zhang, "Intelligent reflecting surface enhanced wireless network via joint active and passive beamforming," IEEE Trans. Wireless Commun., vol. 18, no. 11, pp. 5394-5409, Nov. 2019.

[10] E. Björnson, Ö. Özdogan, and E. G. Larsson, "Intelligent reflecting surface vs. decode-and-forward: How large surfaces are needed to beat relaying?" IEEE Wireless Commun. Lett., 2019, to appear.

[11] C. A. Balanis, Advanced Engineering Electromagnetics, 2nd ed. John Wiley \& Sons, 2012.

[12] N. Yu, P. Genevet, M. A. Kats, F. Aieta, J.-P. Tetienne, F. Capasso, and Z. Gaburro, "Light propagation with phase discontinuities: Generalized laws of reflection and refraction," Science, vol. 334, no. 6054, pp. 333337, 2011

[13] V. S. Asadchy, M. Albooyeh, S. N. Tcvetkova, A. Díaz-Rubio, Y. Ra'di, and S. A. Tretyakov, "Perfect control of reflection and refraction using spatially dispersive metasurfaces," Phys. Rev. B, vol. 94, p. 075142, Aug 2016.

[14] D. Headland, T. Niu, E. Carrasco, D. Abbott, S. Sriram, M. Bhaskaran, C. Fumeaux, and W. Withayachumnankul, "Terahertz reflectarrays and nonuniform metasurfaces," IEEE Journal of Selected Topics in Quantum Electronics, vol. 23, no. 4, July 2017.

[15] N. M. Estakhri and A. Alú, "Wave-front transformation with gradient metasurfaces," Phys. Rev. X, vol. 6, p. 041008, Oct. 2016.

\footnotetext{
${ }^{3}$ More specifically, 5 Sec. II.D] states that "a metasurface that is capable of shaping the angle and the phase of the reflected signal has a size of the order of $10 \lambda \times 10 \lambda$. This size allows, in general, a metasurface to be viewed as a specular reflector according to geometrical optics." On the contrary, as shown in Fig. 2 herein, the beamwidth of the scattered wave from such a surface is $\approx 10^{\circ}$, hence it is far from a specular reflector, irrespective of $\lambda$, when the source and destination are in the far-field.
} 